\title{
ON THE SINGULARITIES OF CONTINUOUS LEGENDRE TRANSFORMS
}

\author{
GILBERT G. WALTER AND AHMED I. ZAYED
}

\begin{abstract}
The analytic properties of continuous Legendre transform $F(\lambda)$ of a function $f(t)$ holomorphic in an elliptical neighborhood of the real interval $[-1,1]$ are investigated. It is shown to be an entire function of exponential type whose Borel transform $g(z)$ has a singularity at $z_{0}$ if and only if $f(t)$ has one at $t_{0}$ where $z_{0}=\cosh t_{0}$. The proof involves a modification of "Hadamard's argument" on multiplication of singularities. The result may also be interpreted as a statement about the second continuous Legendre transform which gives $f(t)$ in terms of $F(\lambda)$.
\end{abstract}

1. Introduction. The problem of locating the singular points of eigenfunction expansions is closely related to the problem of locating the singularities of solutions of partial differential equations. This in turn is of great importance in both modern physics and applied mathematics (see [5] for references). One of the main techniques used to locate the singularities of a solution of a partial differential equation is to map this solution, usually by means of an integral transform, into an analytic function whose singularities are easier to locate.

One of the earliest results in this direction was obtained by Szegö in [9] where he related the singularities of the harmonic function

$$
f(r, \theta)=\sum_{n=0}^{\infty} a_{n} r^{n} P_{n}(\cos \theta)
$$

where $\overline{\lim }_{n \rightarrow \infty} \sqrt{\left|a_{n}\right|}=1$ and $P_{n}(x)$ is the Legendre polynomial of degree $n$ to the singularities of the analytic function

$$
g(z)=\sum_{n=0}^{\infty} a_{n} z^{n} .
$$

Using what is now known as Hadamard's argument, Nehari [8] was able to extend Szegö's result to the case where

$$
f(t)=\sum_{n=0}^{\infty} a_{n} P_{n}(t)
$$

where $\overline{\lim }_{n \rightarrow \infty} \sqrt[n]{\left|a_{n}\right|}=\zeta<1$ and $t$ is complex.

Later, Nehari's result was in turn generalized in two main directions. In one direction, the Legendre polynomials in (1.2) were replaced by the Gegenbauer polynomials, Jacobi polynomials, and other orthogonal functions $[3,4$ and 6$]$. In the

Received by the editors July 1,1985 . Presented to the Society, at the 92 nd annual meeting, January 1986.

1980 Mathematics Subject Classification. Primary 44A15: Secondary 30B40.

Key words and phrases. Continuous Legendre transforms, singularities, analytic continuation. 
other direction, the restriction on the coefficients $a_{n}$ in (1.2) was relaxed to $a_{n}=O\left(n^{p}\right)$ for some $p$ as $n \rightarrow \infty[10,11]$.

The main aim of this paper is to generalize Nehari's result in a third direction. Namely, we shall find the analogue of Nehari's result in the case where the Legendre polynomials in (1.2) are replaced by the Legendre functions $P_{\lambda}(t) ; \lambda \in[-1 / 2, \infty)$ and consequently the series (1.2) representing $f(t)$ is replaced by an integral. More precisely, we will locate the singularities of the function $f(t)$ given by

$$
f(t)=4 \int_{0}^{\infty} F(\lambda) P_{\lambda-1 / 2}(-t) \lambda \sin \pi \lambda d \lambda
$$

by relating them to the singularities of the function

$$
g(z)=\int_{0}^{\infty} e^{-z \lambda} F(\lambda) d \lambda
$$

where

$$
F(\lambda)=\frac{1}{2} \int_{-1}^{1} f(t) P_{\lambda-1 / 2}(t) d t
$$

is assumed to satisfy certain growth conditions at $\infty$.

The main idea of the proof is to construct two integral operators; one to map $f$ into $g$ and the other to map $g$ into $f$ and then employ Hadamard's argument to relate the singularities of $f$ to those of $g$. Furthermore, if it satisfies $(1.5), F(\lambda)$ will be an entire function of exponential type whose Borel transform is the function $g(z)$. Hence the same proof enables us to locate the indicator diagram of $F(\lambda)$ from these same singularities.

2. Preliminaries. The Legendre functions $P_{\lambda}(z)$ are defined for $\lambda \in \mathbf{R}$ by

$$
P_{\lambda}(z)={ }_{2} F_{1}(-\lambda, \lambda+1,1 ;(1-z) / 2) \text {. }
$$

Since $P_{-\lambda}(z)=P_{\lambda-1}(z)$ it suffices to consider the case $\lambda \geqslant-1 / 2$.

We list some properties of the Legendre functions that will be used in the sequel and refer the reader to [1 and 2] for the proofs:

$$
\begin{gathered}
\frac{d}{d x}\left[\left(x^{2}-1\right) P_{\lambda}^{\prime}(x)\right]=\lambda(\lambda+1) P_{\lambda}(x), \\
(2 \lambda+1) z P_{\lambda}(z)=(\lambda+1) P_{\lambda+1}(z)+(\lambda) P_{\lambda-1}(z), \\
P_{\lambda}(z)=O\left(|\zeta|^{\lambda}\right) \quad \text { as } \lambda \rightarrow \infty \text { where } z=\frac{1}{2}\left(\zeta+\zeta^{-1}\right), \\
\int_{-1}^{1} P_{\lambda}(x) P_{\nu}(-x) d x=2 \frac{\sin \pi \lambda-\sin \pi \nu}{\pi(\lambda-\nu)(\lambda+\nu+1)} .
\end{gathered}
$$

For $f(x) \in L^{2}(-1,1)$ the first continuous Legendre transform of $f(x)$ is defined by (1.5), and if $\sqrt{\lambda} F(\lambda) \in L^{1}(0, \infty)$, the second continuous Legendre transform is defined by (1.3). The two are inverses of each other [1].

Before we study the singularities of the function $f(t)$ and the growth of $F(\lambda)$ we need a few basic properties of the latter. Butzer et al. [1] have shown that 
$F(\lambda) \in L^{p} \cap C_{0}$ for $p>2$ and $F(\lambda)=O\left(\lambda^{-1 / 2}\right)$ as $\lambda \rightarrow \infty$ whenever $f \in$ $L^{2}(-1,1)$. However, slightly stronger results are obtained as byproducts of the following theorem:

THEOREM 2.1. Let $f \in L^{2}(-1,1)$ and

$$
F(\lambda)=\hat{f}(\lambda)=\frac{1}{2} \int_{-1}^{1} f(x) P_{\lambda-1 / 2}(x) d x
$$

be its continuous Legendre transform. Then, for real $f$,

(i) $\sqrt{\lambda} F(\lambda) \in L^{2}\left(\mathbf{R}^{+}\right)$,

(ii) $\|f\|^{2} \geqslant 2 \int_{0}^{\infty} \lambda F^{2}(\lambda) d \lambda$,

(iii) $F(\lambda)$ is entire in $\lambda$ of exponential type $\leqslant \pi$.

Proof. The discrete Legendre transformation of $f$ is just the set of coefficients

$$
a_{k}=\frac{2 k+1}{2} \int_{-1}^{1} f(x) P_{k}(x) d x
$$

for which we have by Parseval's equality

$$
\sum_{k=0}^{\infty} \frac{\left|a_{k}\right|^{2}}{(k+1 / 2)}=\|f\|_{2}^{2}
$$

By substituting the expansion $f(x)=\sum_{k=0}^{\infty} a_{k} P_{k}(x)$ into (2.6) we have

$$
F(\lambda)=\frac{1}{2} \sum_{k=0}^{\infty} a_{k} \int_{-1}^{1} P_{k}(x) P_{\lambda-1 / 2}(x) d x=\sum_{k=0}^{\infty} a_{k} \hat{P}_{k}(\lambda-1 / 2)
$$

where the convergence is uniform on compact subsets of $\mathbf{R}^{+}$. Let us consider the integral $I_{R}$ given by

$$
\begin{aligned}
I_{R} & =\int_{0}^{R} \lambda F^{2}(\lambda) d \lambda=\int_{0}^{R} \lambda \sum_{k=0}^{\infty} a_{k} \hat{P}_{k}\left(\lambda-\frac{1}{2}\right) \sum_{j=0}^{\infty} a_{j} \hat{P}_{j}\left(\lambda-\frac{1}{2}\right) d \lambda \\
& =\sum_{k=0}^{\infty} \sum_{j=0}^{\infty} a_{k} a_{j} I_{k, j, R}
\end{aligned}
$$

where

$$
I_{k, j, R}=\int_{0}^{R} \lambda \hat{P}_{k}\left(\lambda-\frac{1}{2}\right) \hat{P}_{j}\left(\lambda-\frac{1}{2}\right) d \lambda
$$

But from equation (2.18) in [1] we obtain

$$
\hat{P}_{k}\left(\lambda-\frac{1}{2}\right)=\frac{(-1)^{k} \sin \pi\left(\lambda-\frac{1}{2}\right)}{\pi\left(\lambda^{2}-\left(k+\frac{1}{2}\right)^{2}\right)} .
$$


Therefore (2.10) is given by

$$
\begin{aligned}
I_{k, j, R}= & \frac{(-1)^{k+j}}{\pi^{2}} \int_{0}^{R} \frac{\lambda \sin ^{2} \pi\left(\lambda-\frac{1}{2}\right)}{\left[\lambda^{2}-\left(k+\frac{1}{2}\right)^{2}\right]\left[\lambda^{2}-\left(j+\frac{1}{2}\right)^{2}\right]} d \lambda \\
= & \frac{(-1)^{k+j}}{\pi^{2}(k+j+1)} \int_{0}^{R}\left[\frac{\sin ^{2} \pi\left(\lambda-\frac{1}{2}\right)}{\left(\lambda-k-\frac{1}{2}\right)\left(\lambda-j-\frac{1}{2}\right)}\right. \\
& \left.-\frac{\sin ^{2} \pi\left(\lambda-\frac{1}{2}\right)}{\left(\lambda+k+\frac{1}{2}\right)\left(\lambda+j+\frac{1}{2}\right)}\right] d \lambda \\
= & \frac{(-1)^{k+j}}{\pi^{2}(k+j+1)} \int_{-R}^{R} \frac{\cos ^{2} \pi \lambda}{\left(\lambda-k-\frac{1}{2}\right)\left(\lambda-j-\frac{1}{2}\right)} \operatorname{sgn} \lambda d \lambda .
\end{aligned}
$$

For $k=j$, we have

$$
\begin{aligned}
\pi^{2}(2 k+1) \tilde{I}_{k} & =\pi^{2}(2 k+1) \lim _{R \rightarrow \infty} I_{k, k, R}=\int_{-\infty}^{\infty} \frac{\cos ^{2} \pi \lambda \operatorname{sgn} \lambda}{\left(\lambda-k-\frac{1}{2}\right)^{2}} d \lambda \\
& =\int_{-\infty}^{\infty} \frac{\sin ^{2} \pi \lambda \operatorname{sgn}\left(\lambda+k+\frac{1}{2}\right)}{\lambda^{2}} d \lambda=\int_{-k-\frac{1}{2}}^{k+\frac{1}{2}} \frac{\sin ^{2} \pi \lambda}{\lambda^{2}} d \lambda \\
& =2 \int_{0}^{k+\frac{1}{2}} \frac{\sin ^{2} \pi \lambda}{\lambda^{2}} d \lambda,
\end{aligned}
$$

and thus,

$$
\begin{aligned}
\left|\tilde{I}_{k}\right| & \leqslant \frac{1}{\pi^{2}\left(k+\frac{1}{2}\right)}\left|\int_{0}^{k+\frac{1}{2}} \frac{\sin ^{2} \pi \lambda}{\lambda^{2}} d \lambda\right| \\
& \leqslant \frac{1}{\pi^{2}\left(k+\frac{1}{2}\right)} \int_{0}^{\infty} \frac{\sin ^{2} \pi \lambda}{\lambda^{2}} d \lambda \leqslant \frac{1}{(2 k+1)} .
\end{aligned}
$$

For $k \neq j$, we use Schwarz's inequality to deduce that

$$
I_{k, j}=\lim _{R \rightarrow \infty} I_{k, j, R} \leqslant\left(\tilde{I}_{k}\right)^{1 / 2}\left(\tilde{I}_{j}\right)^{1 / 2} \leqslant\left(\frac{1}{2 k+1}\right)^{1 / 2}\left(\frac{1}{2 j+1}\right)^{1 / 2}
$$

Hence, by combining (2.9), (2.10), (2.13) and (2.14) we have

$$
I_{R} \leqslant \sum_{k=0}^{\infty} \sum_{j=0}^{\infty}\left|a_{k}\left\|a_{j} \mid\left(\tilde{I}_{k}\right)^{1 / 2}\left(\tilde{I}_{j}\right)^{1 / 2} \leqslant \sum_{k=0}^{\infty} \frac{a_{k}^{2}}{(2 k+1)}=\frac{1}{2}\right\| f \|_{2}^{2},\right.
$$

and the first two conclusions follow from (2.15) by taking the limit as $R \rightarrow \infty$.

To prove (iii) it follows that $F(\lambda)$ is entire from (2.6), (2.1) and the fact that the hypergeometric function is an entire function in its parameters. If we write $\lambda=\mu+$ 
$i \nu$, then from (2.8) and (2.11) we obtain

(2.16)

$$
\begin{aligned}
|F(\mu+i \nu)| \leqslant & \frac{1}{\pi} \sum_{k=0}^{\infty}\left|a_{k}\right|\left|\frac{\sin \pi\left(\lambda-\frac{1}{2}\right)}{\lambda^{2}-\left(k+\frac{1}{2}\right)^{2}}\right| \\
\leqslant & \frac{1}{\pi} \sum_{k=0}^{[\mu]} \frac{\left|a_{k}\right| e^{\pi|\nu|}}{\left|\mu+i \nu-\left(k+\frac{1}{2}\right)\right|\left|\mu+i \nu+k+\frac{1}{2}\right|} \\
& +\frac{1}{\pi} \sum_{k=[\mu]+1}^{\infty} \frac{\left|a_{k}\right| e^{\pi|\nu|}}{\left|\mu^{2}-\left(k+\frac{1}{2}\right)^{2}\right|} \\
\leqslant & \frac{e^{\pi|\nu|}}{\pi}\left(\sum_{k=0}^{[\mu]} \frac{\sqrt{k+\frac{1}{2}}}{|\nu|^{2}}+\sum_{k=0}^{\infty} \frac{\sqrt{k+\mu+2}}{\left(k+\frac{1}{2}\right)^{2}}\right)\|f\|_{2} \leqslant C e^{\pi|\lambda|} \text {. Q.E.D. }
\end{aligned}
$$

3. Integral representations. In the last section we assumed only that $f(x) \in$ $L^{2}(-1,1)$. In this section, we further assume that $f(z)$ is holomorphic inside the ellipse $\mathscr{G}_{\rho}=\left\{z:\left|z+\left(z^{2}-1\right)^{1 / 2}\right|<\rho ; \rho>1\right\}$ with foci at \pm 1 . Then, we will show that $F(\lambda)$ is not only entire of exponential type but also rapidly decreasing on the real axis.

Let

$$
g(t)=\int_{0}^{\infty} e^{-t \lambda} F(\lambda) d \lambda
$$

be the Borel transform of $F(\lambda)$ where the integral is taken over a ray emanating from the origin. It is known [7] that the inveision formula for (3.1) is given by

$$
F(\lambda)=\frac{1}{2 \pi i} \oint_{\gamma} e^{i \lambda} g(t) d t
$$

where $\gamma$ is any contour enclosing the conjugate diagram of $F(\lambda)$ which is the smallest convex domain containing the singularities of $g(t)$.

Now we construct two integral operators, one to map $f(z)$ into $g(t)$ and the other to map $g(t)$ into $f(z)$. From (3.1) and (2.6) we have

$$
\begin{aligned}
g(t) & =\int_{0}^{\infty} e^{-t \lambda} \frac{1}{2} \int_{-1}^{1} f(z) P_{\lambda-1 / 2}(z) d z d \lambda \\
& =\int_{-1}^{1} f(z) K(t, z) d z,
\end{aligned}
$$

where

$$
K(t, z)=\frac{1}{2} \int_{0}^{\infty} e^{-t \lambda} P_{\lambda-1 / 2}(z) d \lambda .
$$

On the other hand, from (1.3) and (3.2) we have

$$
\begin{aligned}
f(z) & =4 \int_{0}^{\infty} \frac{1}{2 \pi i} \oint_{\gamma} e^{t \lambda} g(t) d t P_{\lambda-1 / 2}(-z) \lambda \sin \pi \lambda d \lambda \\
& =\oint_{\gamma} g(t) L(t, z) d t,
\end{aligned}
$$


where

$$
L(t, z)=\frac{2}{\pi i} \int_{0}^{\infty} e^{i \lambda} P_{\lambda-1 / 2}(-z) \lambda \sin \pi \lambda d \lambda .
$$

Interchanging the integrals is valid in (3.3) provided at $\operatorname{Re} t$ is sufficiently large and in (3.5) provided that all the singularities of $g(t)$ lie in $\operatorname{Re} t \leqslant-\delta ; \delta>0$. This is indeed the case as is shown in Proposition 3.1.

LEMMA 3.1. Let $f(z)$ be holomorphic in the interior of the ellipse $\mathscr{G}_{\rho}$ where $\rho>1$. Then

$$
F(\lambda)=O\left((\rho-\varepsilon)^{-\lambda}\right), \quad \lambda \in \mathbf{R}^{+}
$$

Proof. Consider $Q_{\lambda-1 / 2}(z)$ the Legendre function of the second kind given by

$$
Q_{\lambda-1 / 2}(z)=\frac{1}{2} \int_{-1}^{1} \frac{P_{\lambda-1 / 2}(t)}{z-t} d t, \quad \operatorname{Im} z \neq 0 .
$$

Then

$$
\begin{aligned}
F(\lambda) & =\frac{1}{2} \int_{-1}^{1} P_{\lambda-1 / 2}(t) f(t) d t=\frac{1}{2} \int_{-1}^{1} P_{\lambda-1 / 2}(t) \frac{1}{2 \pi i} \oint_{\gamma} \frac{f(z)}{z-t} d z d t \\
& =\frac{1}{2 \pi i} \oint_{\gamma} f(z) \frac{1}{2} \int_{-1}^{1} \frac{P_{\lambda-1 / 2}(t)}{z-t} d t d z=\frac{1}{2 \pi i} \oint_{\gamma} f(z) Q_{\lambda-1 / 2}(z) d z
\end{aligned}
$$

where $\gamma$ is the contour $|\zeta|=\rho-\varepsilon$, where $z=\left(\zeta+\zeta^{-1}\right) / 2$.

$$
Q_{\lambda-1 / 2}(z)=O\left(|\zeta|^{-\lambda}\right) \text { as } \lambda \rightarrow \infty \quad[2],
$$

it follows from (3.9) and (3.10) that

$$
|F(\lambda)| \leqslant \frac{1}{2 \pi} \oint_{\gamma}\left|f(z) \| Q_{\lambda-1 / 2}(z)\right||d z| \leqslant C \max _{|\zeta| \leqslant \rho-\varepsilon}|f(z)|(\rho-\varepsilon)^{-\lambda+1 / 2},
$$

which implies (3.7). Q.E.D.

Proposition 3.1. $g(t)$ is holomorphic in the half-plane $\operatorname{Re} t>-\log \rho, \rho>1$.

Proof. This follows from Lemma 3.1 and the uniform convergence of (3.1) in $\operatorname{Re} t \geqslant-\log \rho+\delta$.

COROLlaRY 3.1. Let $f(z)$ satisfy the hypothesis of Lemma 3.1. Then it has the representation (3.5). Moreover, the contour $\gamma$ in (3.5) can be taken in such a way that it lies entirely in the region $\operatorname{Re} t \leqslant-\delta$, for some $\delta>0$.

4. Singularities of the kernels. Let $K(t, z)$ and $L(t, z)$ be the kernel functions given by (3.4) and (3.6), respectively. From Lemma 3.1, Proposition 3.1 and Corollary 3.1 it follows that $K(t, z)$ defines a holomorphic function in the region $\{z$ : $\left.z \in \mathscr{G}_{\rho}\right\} \times\{t: \operatorname{Re} t>\log \rho\}$ and $L(t, z)$ defines a holomorphic function in the region $\left\{z: z \in \mathscr{G}_{\rho}\right\} \times\{t: \operatorname{Re} t<-\log \rho\}$.

We wish to continue both kernels analytically and find their singular points. 
LEMMA 4.1. The kernel $J(t, z)=\int_{0}^{\infty} e^{-t \lambda} P_{\lambda}(z) d \lambda$ satisfies the differential equation

$$
\frac{d}{d t}(J(t, z) h(t, z))=\frac{\phi(t, z)}{h(t, z)},
$$

where $h(t, z)=\left(1-2 z e^{-t}+e^{-2 t}\right)^{1 / 2}$ and $\phi(t, z)$ is holomorphic for all $t$ and $z$ in the cut-plane $\mathbf{C}-[1, \infty)$.

Proof. Fix $z$ and denote $J(t, z)$ by $J(t)$. Then

$$
\begin{aligned}
J^{\prime}(t)= & \int_{0}^{\infty}-\lambda e^{-\lambda t} P_{\lambda}(z) d \lambda \\
= & -\int_{0}^{\infty} e^{-\lambda t}(2 \lambda-1) z P_{\lambda-1}(z) d \lambda \\
& +\int_{0}^{\infty} e^{-\lambda t}(\lambda-1) P_{\lambda-2}(z) d \lambda=I_{1}+I_{2}
\end{aligned}
$$

by (2.3). But by changing the variable in $I_{1}$ and $I_{2}$, we obtain

$$
\begin{aligned}
I_{1} & =-z \int_{-1}^{\infty} e^{-(\mu+1) t}(2 \mu+1) P_{\mu}(z) d \mu \\
& =\left(2 z J^{\prime}(t)-z J(t)\right) e^{-t}-z \int_{-1}^{0}(2 \mu+1) e^{-(\mu+1) t} P_{\mu}(z) d \mu
\end{aligned}
$$

and

$$
\begin{aligned}
I_{2} & =\int_{-2}^{\infty} e^{-(\mu+2) t}(\mu+1) P_{\mu}(z) d \mu \\
& =e^{-2 t}\left(J(t)-J^{\prime}(t)\right)+\int_{-2}^{0} e^{-(\mu+2) t}(\mu+1) P_{\mu}(z) d \mu .
\end{aligned}
$$

By combining (4.2)-(4.4) we obtain

$$
J^{\prime}(t)=\left(2 z J^{\prime}(t)-z J(t)\right) e^{-t}+\left(J(t)-J^{\prime}(t)\right) e^{-2 t}+\phi(t, z),
$$

where

$$
\phi(t, z)=\int_{-2}^{0} e^{-(\mu+2) t}(\mu+1) P_{\mu}(z) d \mu-z \int_{-1}^{0}(2 \mu+1) e^{-(\mu+1) t} P_{\mu}(z) d \mu .
$$

Hence, $J^{\prime}(t)\left(1-2 z e^{-t}+e^{-2 t}\right)+J(t)\left(z e^{-t}-e^{-2 t}\right)=\phi(t, z)$ or

$$
\begin{gathered}
J^{\prime}(t)\left(1-2 z e^{-t}+e^{-2 t}\right)^{1 / 2}+J(t) \frac{\left(z e^{-t}-e^{-2 t}\right)}{\left(1-2 z e^{-t}+e^{-2 t}\right)^{1 / 2}} \\
=\frac{\phi(t, z)}{\left(1-2 z e^{-t}+e^{-2 t}\right)^{1 / 2}}
\end{gathered}
$$

which is equivalent to (4.1). Q.E.D.

THEOREM 4.1. The only possible singularities of $K(t, z)$ and of $L(t, z)$ for nonreal $z$ are at $z=\frac{1}{2}\left(e^{t}+e^{-t}\right)$. 
Proof.

$$
\begin{aligned}
K(t, z) & =\frac{1}{2} \int_{-1 / 2}^{\infty} e^{-(\mu+1 / 2) t} P_{\mu}(z) d \mu \\
& =\frac{1}{2} e^{-t / 2} J(t, z)+\frac{1}{2} \int_{-1 / 2}^{0} e^{-(\mu+1 / 2) t} P_{\mu}(z) d \mu \\
& =\frac{1}{2} e^{-t / 2} J(t, z)+\phi_{1}(t, z)
\end{aligned}
$$

and

$$
\begin{aligned}
L(t, z)= & \frac{2}{\pi i} \int_{1 / 2}^{\infty} e^{t(\mu+1 / 2)} P_{\mu}(-z)(\mu+1 / 2) \cos \pi \mu d \mu \\
= & \frac{2}{\pi i} e^{t / 2} \int_{0}^{\infty}\left(\frac{e^{(t+i \pi) \mu}+e^{(t-i \pi) \mu}}{2}\right) P_{\mu}(-z)(\mu+1 / 2) d \mu \\
& +\frac{2}{\pi i} \int_{-1 / 2}^{0} e^{t(\mu+1 / 2)} P_{\mu}(-z)(\mu+1 / 2) \cos \pi \mu d \mu \\
= & -\left(e^{t / 2} / \pi i\right)\{d J(-t-i \pi,-z) / d t+d J(-t+i \pi,-z) / d t \\
& \left.\quad+\frac{1}{2} J(-t-i \pi,-z)+\frac{1}{2} J(-t+i \pi,-z)\right\}+\phi_{2}(t, z)
\end{aligned}
$$

where $\phi_{1}$ and $\phi_{2}$ are holomorphic in the same region where $\phi$ is holomorphic.

The only possible singularities of $J(t, z)$ are clearly at $z=\frac{1}{2}\left(e^{t}+e^{-t}\right)$. Hence, by (4.5) it follows that these are also the only possible singularities of $K(t, z)$. By (4.6) one can easily see that the only possible singularities of $L(t, z)$ are at $-z$ $=\frac{1}{2}\left(e^{-t \mp i \pi}+e^{t \pm i \pi}\right)=-\frac{1}{2}\left(e^{t}+e^{-t}\right)$. Q.E.D.

5. The singularity theorem. Now we are able to prove our main theorem:

THEOREM 5.1. Let $f(z)$ be holomorphic in the ellipse $\mathscr{G}_{\rho}$. Then

(i) $f(z)$ has a singular point at $z=z_{0}$ if and only if the function $g(t)$ defined by (3.1) has one at $t_{0}$ where

$$
z_{0}=\frac{1}{2}\left(e^{t_{0}}+e^{-t_{0}}\right)
$$

(ii) The continuous Legendre transform $F(\lambda)$ of $f(z)$ is an entire function of exponential type whose conjugate diagram contains a point $\alpha$ if and only if $f(z)$ has a singular point at $\beta$ where $\beta=\frac{1}{2}\left(e^{\alpha}+e^{-\alpha}\right)$.

Proof. (i) The proof is a mere application of Hadamard's argument. We will sketch the proof and refer the reader to [8] for more details. We start with (3.3). The integral defining $g(t)$ can now be taken along a contour $\gamma$ joining the points \pm 1 and lying inside $\mathscr{G}_{\rho}$. As $t$ moves in the $t$-plane, the singularities of $K(t, z)$ move in the $z$-plane and the integral in (3.3) remains unchanged if we can deform $\gamma$ so that no singularity of $K$ can cross over it. This is always possible unless $\gamma$ becomes pinched between a singularity of $f$ and a singularity of $K$, i.e., where $f$ and $K$ have 
a common singular point, say $z_{0}$. This gives rise to a possible singular point of $g(t)$ at $t_{0}$ where $t_{0}$ and $z_{0}$ are related by (5.1). Going in the other direction, we use (3.5) instead and apply the same argument as before to conclude that a common singularity of $g$ and $L$ (say $t_{0}$ ) gives rise to a possible singularity of $f$ at $z_{0}$ where $z_{0}$ and $t_{0}$ are related as before. Thus, by combining the two arguments, we conclude that $f$ has a singularity at $z_{0}$ if and only if $g$ has one at $t_{0}$.

(ii) This follows from (i), (3.2) and Theorem 33 in [7]. Q.E.D.

\section{REFERENCES}

1. P. Butzer, R. Stens and M. Wehrens, The continuous Legendre transform: its inverse transform and applications, Internat. J. Math. Sci. 3 (1980), 47-67.

2. A. Erdelyi, Higher transcendental functions, vol. 1, McGraw-Hill, New York, 1953.

3. R. Gilbert, Integral operator methods in bi-axially symmetric potential theory, Contrib. Differential Equations 2 (1963), 441-456.

4. B_ Bergman's integral operator method in generalized axially symmetric potential theory, J. Math. Phys. 5 (1964), 983-997.

5. __ Function theoretic methods in partial differential equations, Academic Press, New York, 1969.

6. R. Gilbert and H. Howard, A generalization of a theorem of Nehari, Bull. Amer. Math. Soc. 72 (1966), 37-43.

7. B. Ja. Levin, Distribution of zeros of entire functions, Trans. Math. Monographs, vol. 5, Amer. Math. Soc., Providence, R.I., 1964.

8. Z. Nehari, On the singularities of Legendre expansions, J. Rational Mech. Anal. 5 (1956), 987-992.

9. G. Szegö, On the singularities of zonal harmonic expansions, J. Rational Mech. Anal. 3 (1954), $561-564$.

10. G. Walter, On real singularities of Legendre expansions, Proc. Amer. Math. Soc. 19 (1968), 1407-1412.

11. A. Zayed, On the singularities of Gegenbauer (ultraspherical) expansions, Trans. Amer. Math. Soc. 262 (1980), 487-503.

Department of Mathematics, University of Wisconsin, Milwaukee, Wisconsin 53201

Department of Mathematics, California Polytechnic State University, San luis Obispo, CALIFORNIA 93407 